\title{
Chapter 7 \\ Barriers to Point of Care Testing in India and South Africa
}

\author{
Nora Engel, Vijayashree Yellappa, Malika Davids, Keertan Dheda, \\ Nitika Pant Pai and Madhukar Pai
}

\subsection{Introduction}

Point of care (POC) testing in communities, home settings, and primary healthcare centers is widely believed by the global health community to have tremendous potential in reducing delays in diagnosing and initiating treatment for diseases such as HIV, tuberculosis, syphilis, and malaria. The idea is that testing nearer to the patient, at the point of care, allows for quick diagnosis and further management decisions (referral, follow-up testing or treatment) completed in the same clinical encounter or at least the same day, while the patient waits. In this way, the POC continuum is ensured (Pant Pai et al. 2012). POC testing promises to overcome long turnaround times and delays associated with conventional laboratory-based testing. These problems can result in the loss of patients from testing and treatment pathways with detrimental consequences for the development of advanced disease stages and drug resistance.

\author{
N. Engel ( $\square)$ \\ Maastricht University, Maastricht, Netherlands \\ e-mail: n.engel@maastrichtuniversity.nl \\ V. Yellappa \\ Institute of Public Health, Bangalore, India \\ e-mail: vijayashree@iphindia.org \\ M. Davids · K. Dheda \\ University of Cape Town, Cape Town, South Africa \\ e-mail: malika.davids@uct.ac.za \\ K. Dheda \\ e-mail: keertan.dheda@uct.ac.za \\ N. P. Pai · M. Pai \\ McGill University, Montreal, Canada \\ e-mail: nitika.pai@mcgill.ca \\ M. Pai \\ e-mail: madhukar.pai@mcgill.ca


The devices that lend themselves to such POC testing are often thought to be simple, cheap, and rapid (Mabey et al. 2012), work without access to laboratories, fridges, gloves, biosafety, continuous power supply, or trained staff and meet what the World Health Organization defined as ASSURED criteria (Affordable, sensitive, specific, user-friendly, rapid and robust, equipment free, and delivered). However, the availability of relatively cheap, simple, and rapid tests that can be conducted outside laboratories does not automatically ensure the POC continuum. We know that f.i. malaria rapid tests are often not used or the results not acted upon (Chandler et al. 2012). Similarly, TB tests deployed at POC might require additional infrastructural, financial, and operational support, exhausting resources at the clinic (Clouse et al. 2012), and HIV rapid testing is at times hampered by poor linkages to care (Kranzer et al. 2010). In order to understand the new roles and challenges that medical devices such as POC tests encounter, we need to study diagnostic practices at the POC and how devices are integrated into workflow and patient pathways.

This chapter reviews results from a qualitative research project on barriers to POC testing in South Africa and India. In this project, we aimed at understanding where POC testing is happening and what the main barriers are. Using a framework that envisions POC testing as programs, rather than just tests, across five settings (home, community, peripheral laboratory, clinic, and hospital) (Pant Pai et al. 2012), we examined diagnostic practices across major diseases and actors in homes, clinics, communities, hospitals, and laboratories in South Africa and India. Detailed results per country have been published (Engel et al. 2015a, b, c). Here, we review selected results, discuss them comparatively, and reflect on the implications for medical device design.

\subsubsection{Qualitative Project on Barriers to POC Testing}

Data for this project was collected in semi-structured interviews $(\mathrm{N}=101$ in South Africa, $\mathrm{N}=8$ in India) and focus group discussions ( $N=7$ in South Africa, $N=13$ in India) with doctors, nurses, community health workers, patients, laboratory technicians, policymakers, hospital managers, and diagnostic manufacturers between September 2012 and June 2013 in Durban, Cape Town, and Eastern Cape (South Africa) and Bangalore and Tumkur district (India). Participants were purposively sampled to represent the settings of hospitals, peripheral labs, clinics, communities, and homes in both the public/private sector and rural/urban setting. In the context of conducting interviews and FGDs, we visited labs, clinics, and testing facilities. These three data sources allowed us to triangulate data. The interviews specifically examined diagnostic steps for each major disease occurring in the setting (such as HIV, TB, diabetes, diarrhoeal diseases and hypertension in South Africa and HIV, TB, malaria, hepatitis, syphilis, diabetes, typhoid, and dengue in India) in great detail from ordering a test to acting on a result, including available material and capacities, turnaround times, and referral processes. Additionally, we explored during interviews the challenges that participants encountered when diagnosing, understanding of 
diagnosis, and visions of an ideal test. The focus group discussions focused exclusively on challenges experienced when diagnosing. Interview and focus group discussion guides were piloted and revised during the fieldwork to improve the clarity of questions. All interviews and discussions were held in English (except some of them in Kannada in India) and digitally recorded, and the notetaker wrote down main points raised, nonverbal communication, and setting characteristics. Data analysis was done thematically (Eisenhardt and Graebner 2007), using Nvivo 9 (QSR International).

\subsubsection{Ethics Approval}

The ethics review board of the University of Cape Town, South Africa and McGill University Health Centre (MUHC), Montreal, Canada approved this study. Approvals for interviews and discussions conducted at public primary healthcare facilities were sought from the Provincial Department of Health authorities as necessary. All participants were provided with information sheets explaining the objectives of the study and all signed informed consent forms prior to participation.

\subsection{Results}

In the following, we review selected results per country. This highlights the very different diagnostic eco-systems (see Figs. 7.1 and 7.2) and the distinct set of challenges to diagnosing at POC, as well as the strategies actors employ to overcome these.

\subsubsection{India}

India has a highly fragmented and largely unregulated diagnostic landscape. Laboratory-based testing takes place across a multitude of providers ranging from small, ill-equipped one-room laboratories in public clinics to large hospital laboratories, from small private neighborhood laboratories with limited testing equipment to medium-sized facilities and state-of-the-art laboratory chains. Patients carry the main responsibility for ensuring a POC continuum. Patients in India are the carriers of samples, reports, and communication between the providers. If they are asked to obtain a diagnostic test in any of the settings, they need to go to the laboratory themselves, provide a sample there, pick up results once available, and return them to the doctor. The system thus relies heavily on patients' initiative to ensure successful POC testing. Oftentimes, these journeys start in the private sector (for rich and poor patients alike) and if patients are not diagnosed and treated successfully, these 


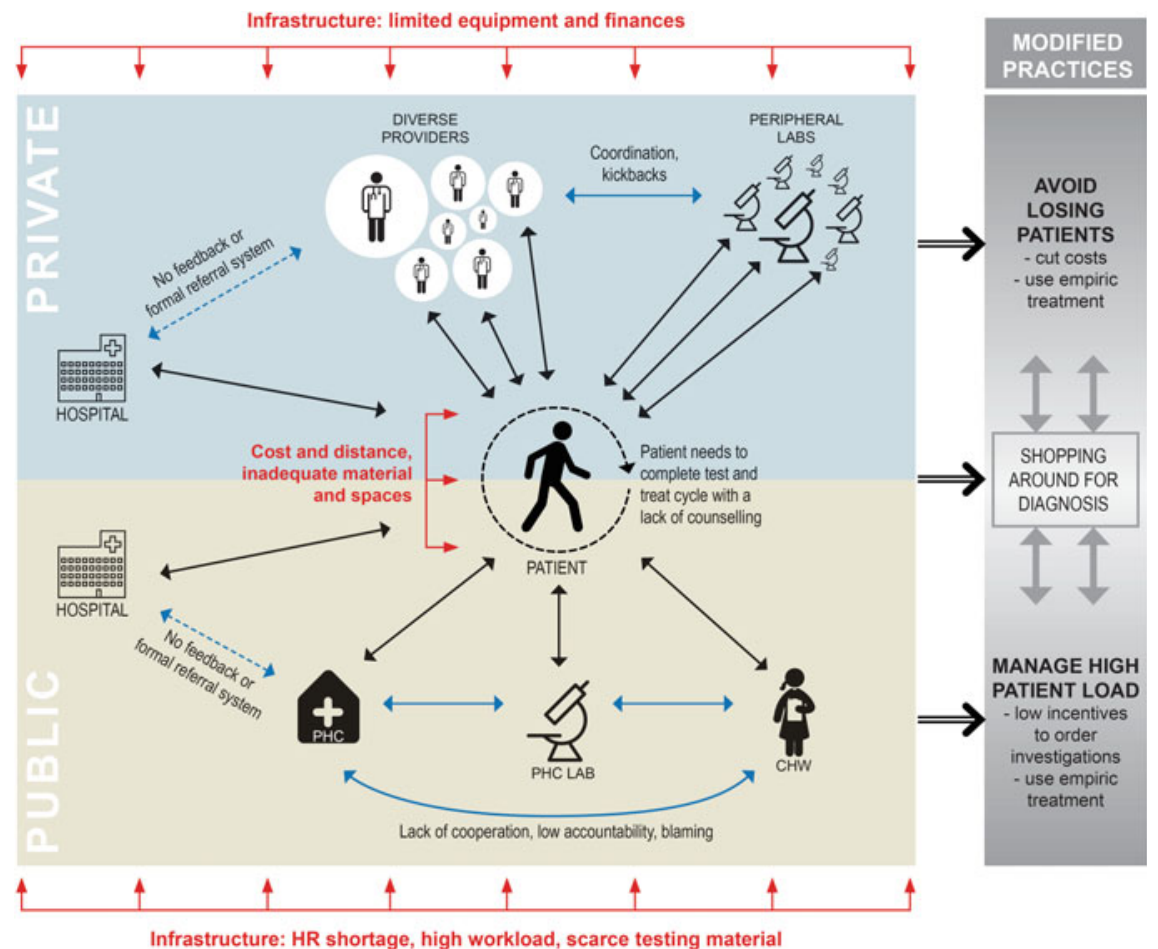

Fig. 7.1 Diagnostic pathway and challenges to POC testing in India (figure first published in Engel et al. 2015b)

journeys can be long, frustrating, expensive, and exhausting or confusing and then chances are high that patients opt out.

Most private practitioners do not conduct any tests on the spot but send their patients to small peripheral laboratories nearby, with whom they have aligned their opening hours and established so-called kickbacks for referring patients (laboratories pay practitioners $30-40 \%$ of the price for each test ordered). These small peripheral laboratories offer a variety of tests including HB card method, random blood sugar (glucometer or manual), urine dipstick or chemical analyser, platelets, complete blood count (CBC), malaria using microscope (rarely rapid card test), blood grouping, HBsAG (hepatitis), VDRL card test (syphilis), typhoid slide (Widal), TB Mantoux, and HIV Tridot. They usually cannot afford rapid test kits and their reagents. But due to smaller volumes (10-30 patients a day), these laboratories are able to maintain a one-hour turnaround time using older methods (Engel et al. 2015c). We showed that thanks to this coordination, a patient seeing a doctor in the morning, can get tested by a nearby lab and return the results to the treating doctor in the afternoon or evening. The coordination thus ensures a POC continuum. Yet, kickbacks can also cause malpractice and a patient's knowledge of these arrangements can lead to 


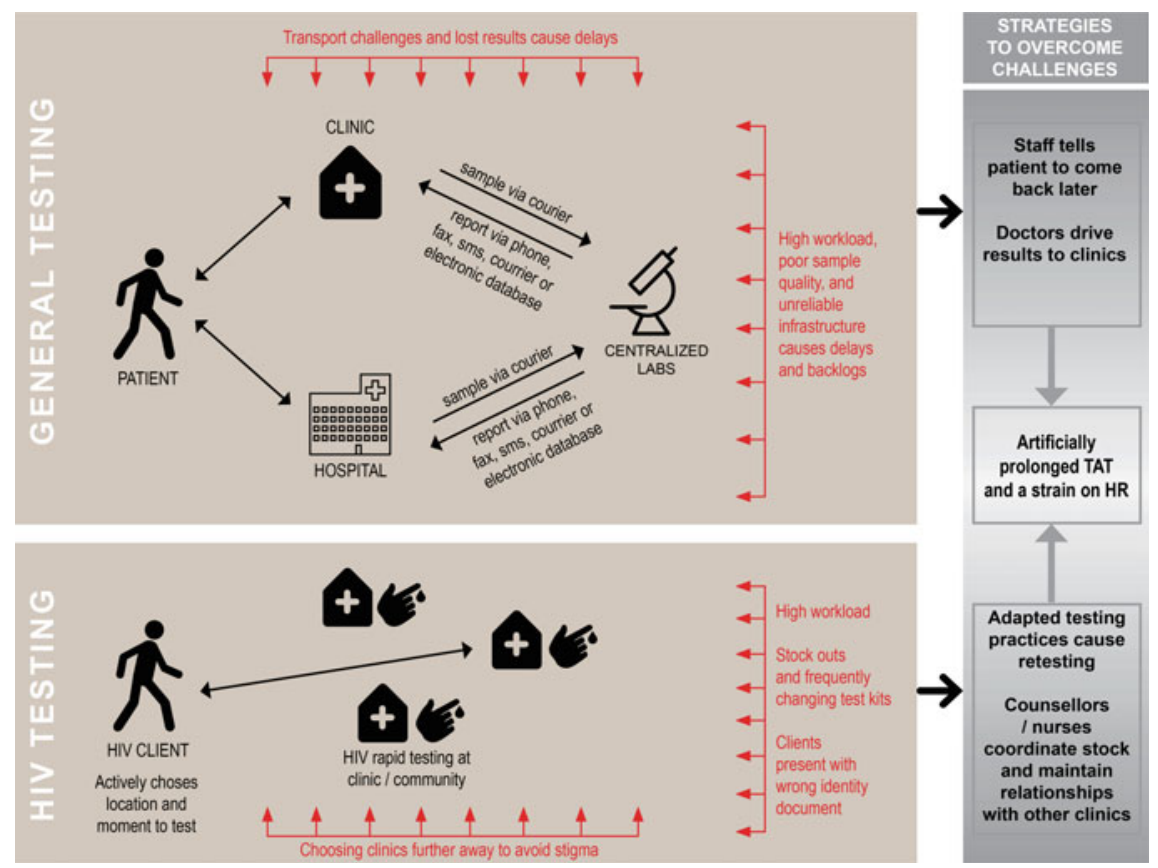

Fig. 7.2 Diagnostic pathway and challenges to POC testing in South Africa

distrust into the care provided, causing patients to change providers and seek care elsewhere (Engel et al. 2015b).

In the public sector, primary public health centers are usually equipped with a small laboratory staffed with a laboratory technician who can run basic tests, such as malaria smears, HbsAg card test (hepatitis), HIV rapid and Coomb's tests, dengue NS1 card test and dengue through IgG and IgM lab tests, urine dipstick, urine sugar testing with Benedict's solution, urine albumin, and total and differential count of white blood cells. However, these laboratories often suffer from underfunding, weak infrastructure, limited budgets for reagents and test kits, and high workloads leading to delays in turnaround times (Engel et al. 2015c). This means that patients are told to come back the next day for results of basic investigations or that they need to be referred for testing to the (sub)-district hospital further away. It also means that medical officers are more inclined to start treating empirically than based on diagnostic test results. Medical officers in public health centers can see up to 100-150 patients per day and lack the time to order investigations or are hesitant due to expected delays or absent materials in the laboratory. If patients accessing these centers are told to come back the next day for results or are referred to another hospital, cost for testing (including potential user fees, transport (from home, to clinic, to diagnostic centers), food and/or accommodation, drugs, and often loss of 
daily wages) increases and the patients might not be able to afford coming back (Engel et al. 2015b).

Loss from diagnostic and treatment pathways can also happen within hospitals. Patients who are asked to get a TB and HIV test done in a hospital outpatient department, for instance, need to queue, provide samples, and return to pick up results in two different laboratories across the hospital compound. While hospital laboratories make use of rapid tests, turnaround times are usually half a day. If the patient came in the morning, he/she needs to find the doctor again after collecting results from the laboratories. The doctor might have left by that time or the outpatient department is closed and they are asked to come back the next day.

Providers suggested centralizing laboratories in hospitals compounds and improving interdisciplinary collaboration among public health center staff. Community health workers suggested that testing at the doorstep could help strengthen trust into the public health clinics among potential patients. However, they also emphasized that testing would have to be followed up with basic treatment and counseling at the doorstep too, since absent drugs, follow-up tests, or doctors at the clinics, as well as irregularly supplied tests to conduct at the doorstep, would spoil that renewed trust again (Engel et al. 2015b).

\subsubsection{South Africa}

Very different from India, South Africa has a highly centralized diagnostic landscape. Diagnostic testing in the public sector is provided by the National Health Laboratory Service (NHLS), while a handful of large diagnostic companies provide diagnostic services to private providers. This means that clinics and smaller hospitals send the majority of their samples via courier to a centralized laboratory, usually in one of the bigger cities, and results are sent back by courier, Internet, SMS, phone, or fax. A few tests are conducted on the spot in public and private clinics. Among them are basic screening tests (blood pressure, weight, glucose, rapid HB, and urine dipstick) and the HIV rapid tests to establish whether someone is HIV infected. In selected public clinics, the Xpert MTB/RIF, a molecular test that promises to diagnose tuberculosis in $90 \mathrm{~min}$, is being implemented [while the majority of the Xpert instruments have been deployed in centralized NHLS laboratories (Cohen et al. 2014)]. For those tests conducted on the spot, results are available within the same patient encounter. An exception is Xpert MTB/RIF where large numbers of samples lead to backlogs and increase the turnaround time from $90 \mathrm{~min}$ to $24 \mathrm{~h}$ (since only four samples can be run in parallel) (Engel et al. 2015a); in addition, it requires 2,5 extra staff to operate the device (Clouse et al. 2012). Treatment initiation can again lead to delays. In the case of HIV, follow-up testing and counseling sessions mean that treatment is initiated 4-7 days after a positive result was established in clinics (Engel et al. 2015a).

The providers reported delays for those diagnostics that are run in centralized laboratories and that no same-day results were available for those tests. Mainly due to transportation challenges (long distances, poor roads, strikes, bad weather, shortages 
of couriers, and damaged samples), but also due to poor sample quality, lost results, misspelled names, excess workload in laboratories, and break down of computer systems. In anticipation of these delays, doctors and nurses would tell patients to come back even later, would drive results out to clinics by themselves, or fetch results in laboratories. These strategies artificially prolong diagnostic turnaround times and put a strain on scarce available human resources (Engel et al. 2015a).

Counselors and nurses who conduct HIV rapid tests (lateral flow assays using blood samples generated by a finger prick) in clinics, outreach settings, and communities need to maintain relationships to other providers to help each other in overcoming stockouts of test kits and reagents and manage high workloads. This could mean that counselors and nurses would share the number of people who came for testing or that hospital doctors would drive test kits to remote clinics based on a good relationship with the nurse working in that clinic (Engel et al. 2017). Irregular supply of test kits, frequently changing test kit brands with slightly different steps to perform (for instance time to result, amount of buffer added), or efforts to accommodate impatient clients mean that providers described different ways of using the test kits (for instance, how long they waited before reading results of the testing strip). These adaptations in practices and differences in how HIV rapid tests were conducted have unclear implications for outcomes of testing and mean that some providers mistrusted each other's quality of diagnostic work, leading to replication of testing and additional delays (Engel et al. 2017). Some providers suggested to establish comprehensive quality control measures, systems of maintenance, and ensure continuous (re)-training for every new rapid test kit that counselors and nurses use. While some wished more testing could be done at POC to guide on the spot treatment decisions or at doorsteps in communities to reduce fear of social stigma when accessing clinics, others emphasized to instead prioritize improving referral systems to centralized laboratories, staff training, and infrastructure strengthening.

Clients who seek an HIV test actively manage their diagnostic processes including attempts to control where they receive testing (by choosing testing sites that fit considerations of cost, distance, or avoidance of social stigma), how and which results they are able to obtain. This could sometimes mean to present with a wrong identity to be eligible for testing at a different site or to access a different diagnostic device (in this case, POC CD4 testing, an HIV monitoring test that was only available in selected sites to newly diagnosed clients). These strategies burden an already overstretched public health system by using additional testing resources and creating difficulties with follow-up and tracing. However, they also show how patients actively turn the test into a tool to achieve another goal (in this case to access another testing device) (Engel et al. 2017). 


\subsection{Discussion and Conclusion}

Our results show how the specific diagnostic eco-systems of South Africa (largely centralized testing with the prominent exception of HIV screening) and India (largely peripheral testing spread across a multitude of providers) constitute very different conditions for POC testing and how the major challenges to ensuring POC continuums are linked to this difference.

In India, successful POC testing hardly occurs in any of the settings and for any of the diseases. Many of the rapid tests are used in laboratories where either the single patient encounter advantage is not realized or the rapidity is compromised due to human resources, manpower, and equipment shortages. In smaller peripheral private laboratories and private clinics with shorter turnaround times, rapid tests are unavailable or too costly. The onus to follow through diagnostic pathways is on the patients and providers use coordination mechanisms (opening hours, kickbacks) to ensure some form of POC continuum using older technologies. In South Africa, the majority of testing happens in centralized laboratories, where delays are accumulated due to transportation, human resource, and infrastructure challenges. Providers' strategies to deal with associated delays create new problems, such as artificially prolonged turnaround times, strains on human resources, and quality of testing, compounding additional diagnostic and treatment delays. While most tests conducted on the spot can be made to work successfully as POC tests, delays remain with regard to treatment initiation.

In both countries, actors use different strategies to overcome these challenges. These adaptive strategies to make POC testing work are in both countries rather fragile and ad hoc, dependent on locally negotiated solutions, personal commitment, available human resources, and relationships. While some are successful in ensuring a timely diagnosis, others lead to disruptions, unnecessary testing, or delays with at times unclear implications for quality of diagnosis. In India, strategies for coordinating between private providers and laboratories with kickbacks or treating empirically right away are aimed at avoiding losing patients, and in this way ensure some form of POC continuum. However, they also increase chances for malpractices (wrong or unnecessary tests might be ordered, inadequate treatment might be prescribed) and may lead to mistrust from patients into the health system. In South Africa, strategies of dealing with delays associated with centralized testing actually increase diagnostic delays (such as telling patients to come back even later) or put additional strains on the health system (such as doctors delivering results to remote clinics). Testing on the spot, for instance, HIV testing, requires healthcare providers to maintain functioning relationships to other providers to overcome stockouts and excess workload, while their adaptations in conducting HIV rapid tests to continuously changing test kits or patient demands can foster mistrust among providers.

Patients embody very active roles in managing their diagnostic journeys. In India, the system relies almost entirely on the patient to ensure the POC continuum across homes, clinics, labs, and hospitals, amidst a multitude of public and private providers with divergent and often competing practices in settings lacking material, money, and 
human resources. The onus is on the patient to ensure completion of test and treat cycles. If a patient's initiative is not supported in these journeys, chances are high that he/she opts out. Constructive counseling by providers about various aspects of diagnostic tests and processes is necessary but not sufficient. Functioning relationships between providers are equally important (Engel et al. 2015b).

While the system in South Africa does not foresee such an active role for patients in ensuring a POC continuum, it still relies on a patients' ability to return to clinics on another day for either results (in the case of laboratory-based testing) or for followup testing and counseling sessions in the case of HIV. The examples of HIV testing showed that HIV patients also need to actively manage diagnostic processes to make testing fit their personal circumstances and make testing worthwhile for themselves (Engel et al. 2017).

These profound differences between the diagnostic setup, offer very different conditions for POC testing. Although the promises that have been attached to POC testing easily lend themselves to view testing technologies as silver bullets, it matters how diagnostic processes are organized and made to work at POC. The results reveal how the material dimensions of diagnosis, such as the test platform, reagents, and supplies, the actors involved, their relations and the sociocultural context in which testing and diagnosis are happening are invariably interlinked (Engel et al. 2015b). This means that simply focusing on one element, for instance, improving infrastructure or test platforms or relationships, is not sufficient. Those aspects need to be studied and tackled together. The contrasting results from India and South Africa further highlight that the settings and the tests have their own histories, assumptions, practices, and understandings inscribed in them. This means that by implementing tests successfully, both the setting, including its organization of the workflow, workforce, its infrastructure, interaction with patients, and standards, and the tests are being shaped and need to be adapted. Tools and (user) practices are being co-constructed (Oudshoorn and Pinch 2003). Implementing diagnostic tests is thus a dynamic and ongoing process that requires continuous observation, analysis, reflection, iterations, and adaptations of tests and (user)-practices and -settings.

Such insights need to be taken into account when designing POC testing programs and technologies. Test developers, decision-makers, and funders need to account for these ground realities. They need to identify and involve users and various stakeholders in design, evaluation, and implementation processes. However, current global diagnostic design and development practices, research, regulation, and evaluation capacities do not do justice to the dynamic nature of these processes of making diagnostic tests work at POC.

Based on these insights, practitioners, donors, and test developers should make sure to

- Study diagnostic practices at POC and how devices are integrated into workflow and patient pathways before, during and after design and implementation of new products; 
- Tackle jointly the relationships between providers and between patients and providers, infrastructure, testing platforms, and adaptive strategies of dealing with constraints;

- Examine the role, responsibility, and work of patients to ensure a POC continuum;

- Allow continuous observation, analysis, reflection, iterations, and adaptations of tests and (user)-practices and -settings; and

- Develop research capacity to assess and integrate these factors.

\section{References}

Chandler, C. I., Mangham, L., Njei, A. N., Achonduh, O., Mbacham, W. F., \& Wiseman, V. (2012). As a clinician, you are not managing lab results, you are managing the patient: How the enactment of malaria at health facilities in Cameroon compares with new WHO guidelines for the use of malaria tests. Social Science and Medicine, 74, 1528-1535. https://doi.org/10.1016/j.socscimed. 2012.01.025.

Clouse, K., et al. (2012). Implementation of Xpert MTB/RIF for routine point-of-care diagnosis of tuberculosis at the primary care level. South African Medical Journal, 102, 805-807.

Cohen, G. M., Drain, P. K., Noubary, F., Cloete, C., \& Bassett, I. V. (2014). Diagnostic delays and clinical decision-making with centralized Xpert MTB/RIF testing in Durban, South Africa. JAIDS Journal of Acquired Immune Deficiency Syndromes, 67, e88-93. https://doi.org/10.1097/ QAI.0000000000000309.

Eisenhardt, K. M., \& Graebner, M. E. (2007). Theory building from cases: Opportunities and challenges. Academy of Management Journal, 50, 25-32.

Engel, N., Davids, M., Blankvoort, N., Dheda, K., Pant Pai, N., \& Pai, M. (2017). Making HIV testing work at the point of care in South Africa: A qualitative study of diagnostic practices. BMC Health Services Research, 17, 408. https://doi.org/10.1186/s12913-017-2353-6.

Engel, N., Davids, M., Blankvoort, N., Pai, N. P., Dheda, K., \& Pai, M. (2015a). Compounding diagnostic delays: A qualitative study of point-of-care testing in South Africa. Tropical Medicine and International Health, 20, 493-500. https://doi.org/10.1111/tmi.12450.

Engel, N., Ganesh, G., Patil, M., Yellappa, V., Pant Pai, N., Vadnais, C., et al. (2015b). Barriers to Point-of-Care testing in India: Results from qualitative research across different settings, users and major diseases. PLOS ONE, 10, e0135112. https://doi.org/10.1371/journal.pone.0135112.

Engel, N., Ganesh, G., Patil, M., Yellappa, V., Vadnais, C., Pai, N., et al. (2015c). Point-of-care testing in India: missed opportunities to realize the true potential of point-of-care testing programs. BMC Health Services Research, 15, 550.

Kranzer, K., et al. (2010). Linkage to HIV care and antiretroviral therapy in Cape Town, South Africa. PLoS ONE, 5, e13801. https://doi.org/10.1371/journal.pone.0013801.

Mabey, D. C., et al. (2012). Point-of-Care tests to strengthen health systems and save newborn lives: The case of syphilis. PLoS Med, 9, e1001233. https://doi.org/10.1371/journal.pmed.1001233.

Oudshoorn, N., \& Pinch, T. J. (2003). Users and Non-Users Matter. In N. Oudshoorn \& T. J. Pinch (Eds.), How users matter: The co-construction of users and technology (pp. 1-25). London, Cambridge MA: MIT Press.

Pant Pai, N., Vadnais, C., Denkinger, C., Engel, N., \& Pai, M. (2012). Point-of-Care testing for infectious diseases: diversity, complexity, and barriers in low- and middle-income countries. PLoS Medicine, 9, e1001306. https://doi.org/10.1371/journal.pmed.1001306. 
Open Access This chapter is licensed under the terms of the Creative Commons Attribution 4.0 International License (http://creativecommons.org/licenses/by/4.0/), which permits use, sharing, adaptation, distribution and reproduction in any medium or format, as long as you give appropriate credit to the original author(s) and the source, provide a link to the Creative Commons license and indicate if changes were made.

The images or other third party material in this book are included in the book's Creative Commons license, unless indicated otherwise in a credit line to the material. If material is not included in the book's Creative Commons license and your intended use is not permitted by statutory regulation or exceeds the permitted use, you will need to obtain permission directly from the copyright holder. 